\title{
Dual Solution of MHD Stagnation-Point Flow towards a Stretching Surface
}

\author{
T. R. Mahapatra ${ }^{1^{*}}$, S. K. Nandy ${ }^{2}$, A. S. Gupta ${ }^{3}$ \\ ${ }^{1}$ Department of Mathematics, Visva-Bharati, Santiniketan, India; \\ ${ }^{2}$ Department of Mathematics, A.K.P.C Mahavidyalaya, Bengai, Hooghly, India; \\ ${ }^{3}$ Department of Mathematics, Indian Institute of Technology, Kharagpur, India. \\ ${ }^{*}$ E-mail: trmahapatra@yahoo.com \\ Received September 19, 2009; revised November 7, 2009; accepted November 12, 2009
}

\begin{abstract}
The effect of a uniform transverse magnetic field on two-dimensional stagnation-point flow of an incompressible viscous electrically conducting fluid over a stretching surface is investigated when the surface is stretched in its own plane with a velocity proportional to the distance from the stagnation-point. This magnetohydrodynamic (MHD) flow problem is governed by the parameter $b$ representing the ratio of the strain rate of the stagnation-point flow to that of the stretching sheet and the magnetic field parameter $\mathrm{M}$. It is known from a previous paper [9] that if $b>1$, the steady solution to the problem is monotonic increasing and the solution is also unique. But when $0<\mathrm{b}<\mathrm{b}_{\mathrm{c}}$ (where $\mathrm{b}_{\mathrm{c}}(<1)$ depends on $\left.\mathrm{M}\right)$, there exists a dual solution which is non-monotonic in addition to a monotonic decreasing solution. It is found in this paper that bc decreases as $\mathrm{M}$ increases. Numerically it is shown that if $\mathrm{M}>0.23919$, the non-monotonic solution cannot exist and so in this case, the only solution is monotonic decreasing. A stability analysis reveals that when $0<\mathrm{b}<$ $b_{c}$, the solutions along the upper branch corresponding to the monotonic solution are linearly stable while those along the lower branch for the non-monotonic solution are linearly unstable. It is also shown that the decay rate of a disturbance increases with increasing $M$ for the stable solution but the growth rate of instability for the non-monotonic solution decreases with increasing $\mathrm{M}$.
\end{abstract}

Keywords: Dual Solution; Magnetohydrodynamic Stagnation-Point Flow; Stretching Surface; Stability Analysis

\section{Introduction}

Flow of an incompressible viscous fluid over a stretching surface has important bearing on several industrial processes. For instance, in the extrusion of a polymer in a melt-spinning process, the extrudate from the die is usually drawn and simultaneously stretched into a sheet which is then cooled gradually by direct contact with water. The property of the final product depends on the rate of heat transfer at the surface of the sheet. Further the study of the flow of an electrically conducting viscous fluid caused by the deformation of the walls of the vessel containing this fluid in the presence of a magnetic field is of great interest in modern metallurgical and metal-working processes. Crane [1] obtained a similarity solution in closed analytical form for steady two dimensional flow of an incompressible viscous fluid caused solely by the stretching of an elastic sheet which moves in its own plane with a velocity varying linearly with distance from a fixed point. Pavlov [2] gave a similarity solution in exact analytical form of the MHD boundary layer equations for steady two-dimensional flow of an electrically conducting incompressible viscous fluid due to the stretching of a plane elastic surface in its own plane in the presence of a uniform transverse magnetic field. This problem was extended by Chakrabarti and Gupta [3] to include suction or injection at the stretching surface. The temperature distribution in the flow was also found by them in the case of constant surface temperature. Andersson [4] investigated the MHD flow of a viscoelastic fluid past a stretching surface in the presence of a uniform transverse magnetic field.

Recently Chiam [5] studied steady two-dimensional stagnation-point flow of an incompressible viscous fluid towards a stretching surface in the case when the parameter $b$ representing the ratio of the strain rate of the stagnation-point flow to that of the stretching surface is 
equal to unity. By removing this highly restrictive assumption $(b=1)$, Mahapatra and Gupta [6] analyzed the steady two dimensional orthogonal stagnation-point flow of an incompressible viscous fluid towards a stretching surface in the general case $b \neq 1$. They found that the structure of the boundary layer depends crucially on the value of $b$. Temperature distribution in the flow was also obtained by them. Earlier Mahapatra and Gupta [7] investigated the steady two- dimensional orthogonal stagnation-point flow of an incompressible viscous electrically conducting fluid towards a stretching surface, the flow being permeated by a uniform transverse magnetic field. The steady and unsteady equations governing stagnation-point flow of an electrically nonconducting incompressible viscous fluid over a stretching surface along with the existence and uniqueness of the solutions of these equations were studied by Paullet and Weidman [8].

In this paper we investigate the dual solution of steady two-dimensional orthogonal stagnation-point flow of an incompressible viscous electrically conducting fluid towards a stretching surface in the presence of a uniform transverse magnetic field. We provided a mathematical proof in [9] that if $b>1$, then the solution of this problem is monotonic increasing and further this solution is unique. But for $0<\mathrm{b}<1$, it was shown that there exists a dual solution which is non-monotonic in addition to a monotonic decreasing solution. We have found numerically in this paper that this dual solution exists upto a certain value of the magnetic parameter M. A linear stability analysis of these two solutions is also presented.

\section{Flow Analysis}

Consider the steady two-dimensional stagnation point flow of an incompressible viscous and electrically conducting fluid permeated by a uniform transverse magnetic field towards a flat stretching surface coinciding with the plane $\mathrm{y}=0$, the flow being confined to the region $y>0$. Two equal and opposite forces are applied on the stretching surface along $\mathrm{X}$-axis so that the surface is stretched with a velocity proportional to the distance from the stagnation-point which is taken as the origin. This is shown in Figure 1.

Using boundary layer approximations, the equations for steady MHD two-dimensional flow are in usual notation

$$
\begin{gathered}
u \frac{\partial u}{\partial x}+v \frac{\partial u}{\partial y}=-\frac{1}{\rho} \frac{\partial p}{\partial x}+v \frac{\partial^{2} u}{\partial y^{2}}+\frac{1}{\rho}\left(\mathbf{j} \times \mathbf{B}_{0}\right)_{x}, \\
0=-\frac{1}{\rho} \frac{\partial p}{\partial y},
\end{gathered}
$$

where $\mathbf{j}$ is the electric current density, $\mathbf{B}_{\mathbf{0}}$ is the imposed uniform magnetic field (acting along the y-direction). In writing (1), we have neglected the induced magnetic

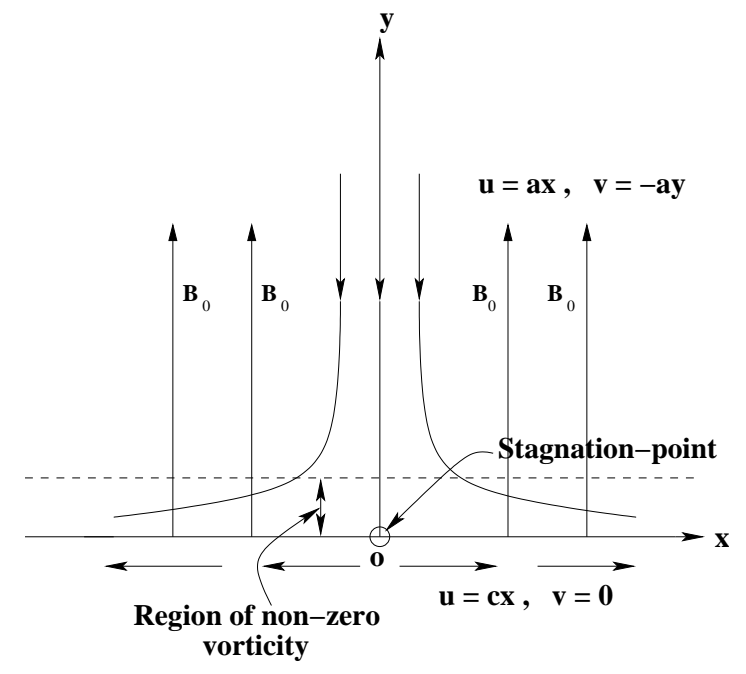

Figure 1. A sketch of the physical problem.

field since the magnetic Reynolds number $R_{M}$ for the flow is assumed to be very small. Such an assumption is justified for the flow of electrically conducting fluids such as liquid metals e.g., liquid sodium, mercury etc. [10].

The equation of continuity is

$$
\frac{\partial u}{\partial x}+\frac{\partial v}{\partial y}=0
$$

Clearly the electric current flows parallel to the z-axis which is normal to the plane of the flow. Hence by Ohm's law

$$
j_{x}=0, j_{y}=0, j_{z}=\sigma\left[E_{z}+u B_{0}\right],
$$

where $\sigma$ is the electrical conductivity of the fluid which is assumed constant and $E_{z}$ is the electric field along $\mathrm{z}$-axis. It is assumed that the current lines in this two-dimensional problem close in a manner consistent with the experiment. Now since the flow is steady, Maxwell's equation gives

$$
\nabla \times \mathrm{E}=0,
$$

where $\mathbf{E}$ is the electric field (which acts parallel to the z-direction). This gives

$$
\frac{\partial E_{z}}{\partial x}=0 \text { and } \frac{\partial E_{z}}{\partial y}=0
$$

so that $\mathrm{E}_{\mathrm{z}}$ is a function of $\mathrm{z}$ only.

Since the induced magnetic field is neglected in view of the assumption $\mathrm{R}_{\mathrm{M}}<<1$, electric current in the flow is determined from Ohm's law and not from $\nabla \times \mathbf{B}=\mu_{e} \mathbf{j}$, $\mu_{\mathrm{e}}$ being the magnetic permeability. But the consequence $\nabla \cdot \mathbf{j}=0$ of this equation must be satisfied [10]. Noting that the physical variables are functions of $x$ and $y$, $\nabla \cdot \mathbf{j}=0$ then gives from (4), $E_{z}=$ constant. Thus using (4), we find from (1), 


$$
\begin{aligned}
u \frac{\partial u}{\partial x}+v \frac{\partial u}{\partial y} & =-\frac{1}{\rho} \frac{\partial p}{\partial x}+v \frac{\partial^{2} u}{\partial y^{2}} \\
& -\frac{\sigma B_{0}{ }^{2} u}{\rho}-\frac{\sigma B_{0} E_{z}}{\rho} .
\end{aligned}
$$

Since from (2), the pressure $\mathrm{p}$ is a function of $\mathrm{x}$ only, the pressure gradient $\frac{\partial p}{\partial x}$ can now be obtained from (6) in the inviscid region as

$$
\frac{1}{\rho} \frac{\partial p}{\partial x}=-U(x) \frac{d U}{d x}-\frac{\sigma B_{0}{ }^{2} U}{\rho}-\frac{\sigma B_{0} E_{z}}{\rho},
$$

where $\mathrm{U}(\mathrm{x})$ is the free stream velocity and use is made of the fact that $E_{z}$ is constant. Hence eliminating $\frac{\partial p}{\partial x}$ from (6) and (7), we get

$$
u \frac{\partial u}{\partial x}+v \frac{\partial u}{\partial y}=U \frac{d U}{d x}+v \frac{\partial^{2} u}{\partial y^{2}}+\frac{\sigma B_{0}^{2}}{\rho}(U-u) .
$$

We note that the electric field does not directly affect the boundary layer equation although it has an influence on the relation between the free stream velocity and the pressure distribution given by (7).

Thus the governing equations for the velocity components $u$ and $v$ are (3) and (8). The boundary conditions are

$$
\begin{gathered}
u=c x, \quad v=0 \quad \text { at } \quad y=0, \\
u \rightarrow U(x)=a x, \quad v \rightarrow-a y \\
\text { as } y \rightarrow \infty
\end{gathered}
$$

where a and c are positive constants. Under the similarity transformation

$$
\begin{aligned}
& u=c x F^{\prime}(\eta), v=-(c v)^{1 / 2} F(\eta), \\
& \eta=y(c / v)^{1 / 2},
\end{aligned}
$$

where a prime denotes derivative with respect to the similarity variable $\eta$, we find that (3) is identically satisfied.

Substitution of (11) in (8) then gives

$$
\begin{aligned}
& F^{\prime \prime \prime}(\eta)+F(\eta) F^{\prime \prime}(\eta)-F^{\prime 2}(\eta) \\
& -M^{2} F^{\prime}(\eta)+M^{2} b+b^{2}=0,
\end{aligned}
$$

where $b=a / c$ and $\mathrm{M}$ is the dimensionless magnetic parameter $B_{0}(\sigma / \rho c)^{1 / 2}$.

The boundary conditions for (12) then follow from (9) and (10) as

$$
F(0)=0, F^{\prime}(0)=1, F^{\prime}(\infty)=b .
$$

A detailed proof of the uniqueness of the solution for the same problem for an electrically non-conducting ( $M$ $=0$ ) fluid was given by Paullet and Weidman [8]. Subsequently the present authors [9] proved the uniqueness of the solution for an electrically conducting fluid in the presence of a magnetic field $(M \neq 0)$ using a similar approach as that in [8]. In [9] we have shown that if $b>1$, the solution of the boundary value problem (BVP) given by (12) and (13) is monotonic increasing for all magnetic parameter $\mathrm{M}$ and further this solution is unique. But if 0 $<\mathrm{b}<1$, there is a dual solution to the above BVP, which is non-monotonic in addition to a solution which is monotonic decreasing. Note that when $b=1$, the above BVP admits of the exact solution $\mathrm{F}(\eta)=\eta$, which is the inviscid solution near the stretching surface as found by Mahapatra and Gupta [7]. However this solution is not frictionless in a strict sense because in this case the friction is uniformly distributed and does not therefore affect the motion.

\section{Numerical Solution}

Since there is no general analytical solution of the non-linear differential equation (12) with the boundary conditions (13) for the case $b \neq 1$, so this system is solved numerically by an efficient shooting method for different values of $M$ and $b$. To do this, we first transform the non-linear differential equation (12) to a system of three first order differential equations as:

$$
\begin{aligned}
& y_{1}^{\prime}=y_{2}, y_{2}{ }^{\prime}=y_{3}, \\
& y_{3}{ }^{\prime}=y_{2}{ }^{2}-y_{1} y_{3}+M^{2}\left(y_{2}-b\right)-b^{2},
\end{aligned}
$$

where $y_{1}=F(\eta), y_{2}=F^{\prime}(\eta), y_{3}=F^{\prime \prime}(\eta)$ and a prime denotes differentiation with respect to the independent variable $\eta$.

The boundary conditions (13) become

$$
\begin{aligned}
& y_{1}=0, y_{2}=1 \text { at } \eta=0, \\
& y_{2} \rightarrow b \text { as } \eta \rightarrow \infty .
\end{aligned}
$$

The values of $\mathrm{y}_{1}$ and $\mathrm{y}_{2}$ are given at the starting point $\eta$ $=0$. But the value of $\mathrm{y}_{2}$ as $\eta \rightarrow \infty$ is replaced by $\mathrm{y}_{2}=\mathrm{b}$ at a finite value $\eta=\eta_{\infty}$ to be determined later. The value of $\mathrm{y}_{3}$ at $\eta=0$ is guessed in order to initiate the integration scheme. Starting from the given values of $\mathrm{y}_{1}$ and $\mathrm{y}_{2}$ at $\eta$ $=0$ and the guessed value of $\mathrm{y}_{3}$ at $\eta=0$, we integrate the first order equations (14) by using a fourth-order Runge-Kutta method up to the end-point $\eta=\eta_{\infty}$. The computed value of $\mathrm{y}_{2}$ at $\eta=\eta_{\infty}$ is then compared with $\mathrm{y}_{2}$ $=b$. The absolute difference between this computed value and $b$ should be as small as possible. To this end we use a Newton-Raphson iteration procedure to assure quadratic convergence of the iterations. The value of $\eta_{\infty}$ is then increased till $\mathrm{y}_{2}$ attains the value $\mathrm{b}$ asymptotically.

\section{Dual Solution of MHD Stagnation-Point Flow}

Our numerical results reveal that for $0<b<1$, in addition to a monotonic decreasing solution which was al- 
ready found by Mahapatra and Gupta [7], there exists a dual solution which is non-monotonic. We have found that in the absence of magnetic field $(M=0)$, the non-monotonic solution exists in the range $0<b \leq$ 0.16906 . This result in the nonmagnetic case $(M=0)$ was also obtained in [8]. For $M=0.1$, the dual solution exists in the range $0<b \leq 0.14845$, and for $M=0.2$, such solution is found in the range $0<b \leq 0.08234$. Numerically we have found that when $M \leq 0.23919$, the nonmonotonic solution exists in the range $0<b<1$. Thus we see that as $M$ increases, the range of $\mathrm{b}$ (such that $0<b<1$ ) for which the dual (i.e., non-monotonic) solution exists progressively decreases. The novel result which emerges from the analysis is that when $M>0.23919$, there is no dual solution in the range $0<b<1$ and the solution in this range is monotonic decreasing and unique.

Figure 2 gives the variation of the dimensionless skin-friction coefficient $F^{\prime \prime}(0)$ with $b$ for several values of $M$. Here the upper branch corresponds to the monotonic solution while the lower branch corresponds to the nonmonotonic one. It can be seen that for the monotonic solution, $\left|F^{\prime \prime}(0)\right|$ increases with increase in $M$. This result can be physically explained as follows. It is clear from the momentum equation (8) that when $b<1$ (i.e., $\mathrm{a}<\mathrm{c}$ ), the Lorentz force given by the last term is negative because the stagnation-point velocity $\mathrm{U}(=\mathrm{ax})$ is less than the stretching velocity $\mathrm{cx}$ of the sheet so that $\mathrm{u}>\mathrm{U}$. Hence this magnetic force decelerates the flow inside the boundary layer. Since the velocity of a fluid particle parallel to the surface is diminished relative to that of the stretching surface, the velocity gradient at the surface increases with increase in the magnetic field $B_{0}$ (characterized by the magnetic parameter $M$ ). This results in increase in the dimensionless skin-friction coefficients $\left|F^{\prime \prime}(0)\right|$ with increase in $M$. Note that the above argument is valid for the monotonic solution for the velocity distribution because in this case the velocity inside the boundary layer decreases monotonically from the surface velocity cx to ax (since $a<c$ ) so that $\mathrm{u}>\mathrm{U}$.

Figures 3-5 display self-similar velocity profiles of $\mathrm{F}^{\prime}(\eta)$ for different values of $M$ at selected values of $b$. The non-monotonic behavior of the velocity distribution is evident from these figures. It is observed from these figures that for a fixed value of $b$, the velocity parallel to the stretching surface at a point in the boundary layer decreases with increase in M. From a physical point of view, this follows from the fact that when $b<1$, increase in $M$ leads to increase in the magnitude of the retarding Lorentz force acting on the fluid resulting in enhanced deceleration of the flow.

An interesting result which emerges from this analysis is that for a given value of $M$, the skin-friction coefficient $\left|\mathrm{F}^{\prime \prime}(0)\right|$ for the dual solution in the case $0<b<1$ is not a monotonic function of $b$ but has a maximum at a particular value of $b$ (see Figure 2). This result is to be contrasted with the corresponding result for the monotonic

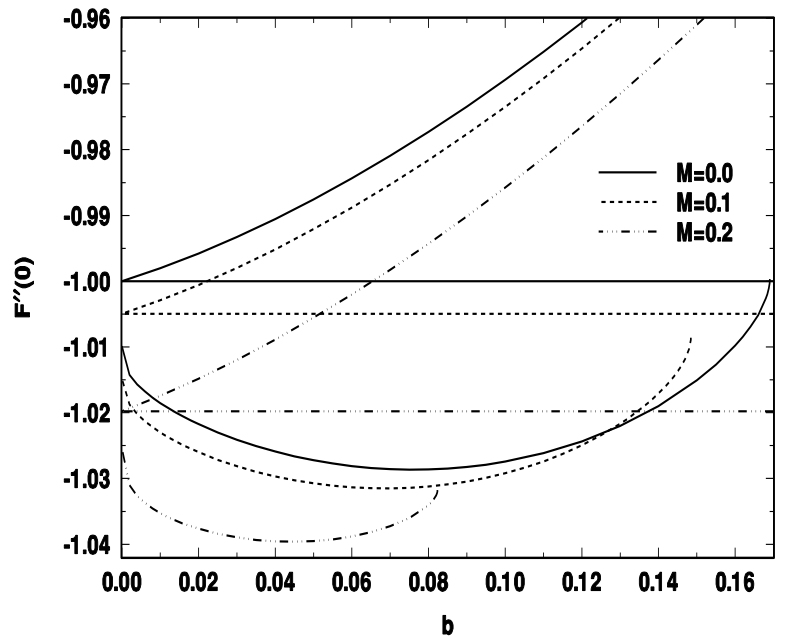

Figure 2. Reduced skin friction coefficient $F^{\prime \prime}(0)$ with $b$ for several values of $M$ showing the upper and lower curves.

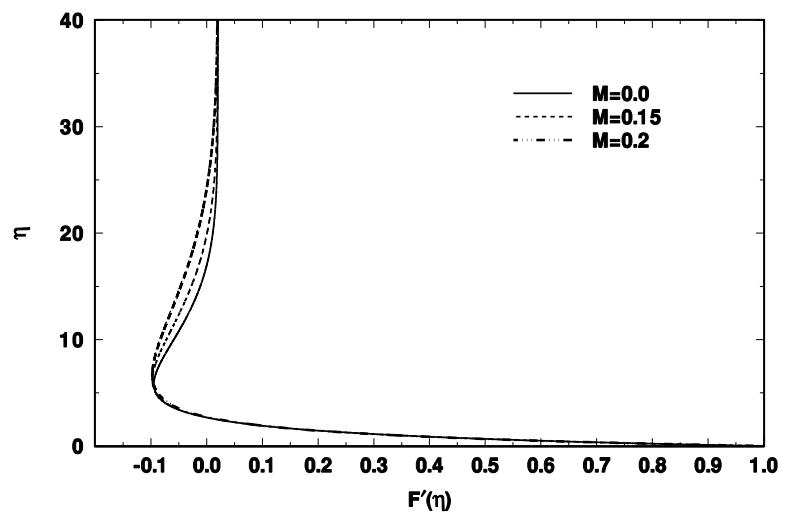

Figure 3. Self-similar velocity profiles (for non-monotonic solution) for $b=0.02$ with different values of $M$.

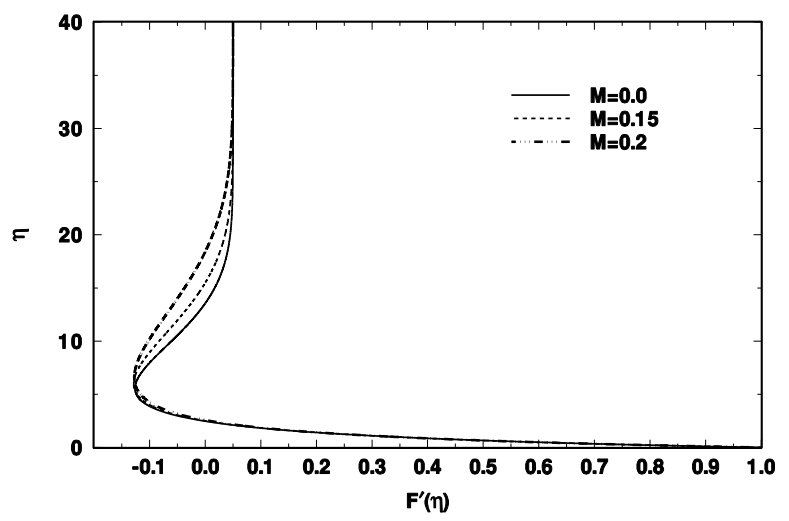

Figure 4. Self -similar velocity profiles (for non-monotonic solution) for $b=0.05$ with different values of $M$.

solution for $0<b<1$, where $\left|\mathrm{F}^{\prime \prime}(0)\right|$ decreases monotonically with increase in $b$ as shown in Figure 2. Physically this stems from the fact that for the dual solution, the 


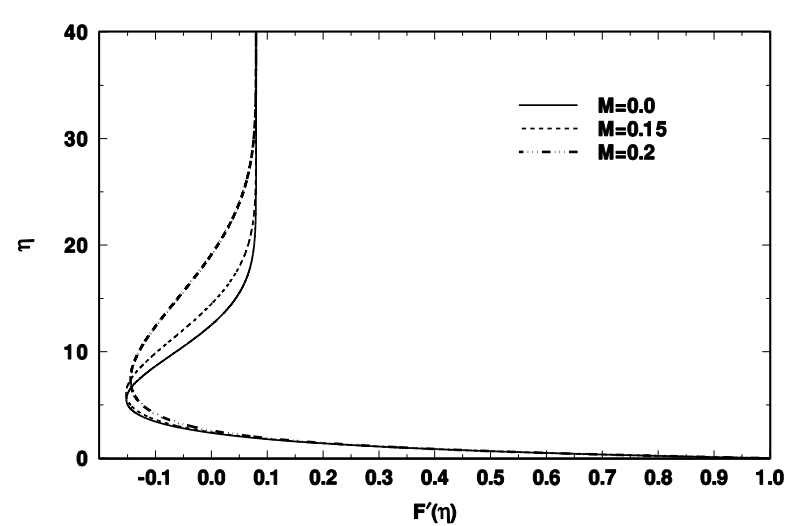

Figure 5. Self -similar velocity profiles (for non-monotonic solution) for $b=0.08$ with different values of $M$.

self-similar velocity profiles $\mathrm{F}^{\prime}(\eta)$ are non-monotonic (see Figures 3-5).

\section{Stability Analysis}

In order to ascertain which of the two solutions in the region $0<b<b_{c}$ (where as shown above, $b_{c}$ depends on $M)$, is expected to appear asymptotically, we test the stability of the above two solutions.

To this end, we consider the unsteady form of the Equation (8) as

$$
\begin{gathered}
\frac{\partial u}{\partial t}+u \frac{\partial u}{\partial x}+v \frac{\partial u}{\partial y}=U \frac{d U}{d x}+v \frac{\partial^{2} u}{\partial y^{2}} \\
+\frac{\sigma B_{0}{ }^{2}}{\rho}(U-u),
\end{gathered}
$$

together with the equation of continuity (3). We seek the unsteady similarity solution in the form

$$
\begin{aligned}
& u=c x f_{\eta}(\eta, \tau), v=-(c v)^{1 / 2} f(\eta, \tau), \\
& \eta=y(c / v)^{1 / 2},
\end{aligned}
$$

where the subscript $\eta$ denotes partial derivative with respect to $\eta$ and $\tau$ is the dimensionless time ct. Note that with $\mathrm{u}$ and $\mathrm{v}$ given above, the equation of continuity (3) is identically satisfied. Substitution of (17) in (16) then gives

$$
\begin{aligned}
& f_{\eta \eta \eta}+f f_{\eta \eta}-f_{\eta}^{2}-M^{2} f_{\eta}+M^{2} b \\
& +b^{2}-f_{\eta \tau}=0,
\end{aligned}
$$

where the subscripts $\eta$ and $\tau$ denote partial derivatives with respect to $\eta$ and $\tau$ respectively. The boundary conditions are

$$
\begin{gathered}
u=c x, \quad v=0 \quad \text { at } \quad y=0, \\
u \rightarrow U(x)=a x, \quad v \rightarrow-a y \\
\text { as } y \rightarrow \infty .
\end{gathered}
$$

In terms of $\mathrm{f}(\eta, \tau)$, these conditions are

$$
f(0, \tau)=0, f_{\eta}(0, \tau)=1, f_{\eta}(\infty, \tau)=b .
$$

Following Merkin [11], we put

$$
f(\eta, \tau)=F(\eta)+e^{-\gamma \tau} g(\eta),
$$

where $\mathrm{F}(\eta)$ satisfies (12) subject to (13) and corresponds to the steady state solution. Further $\mathrm{g}(\eta)$ and all its derivatives are assumed small compared with the steady solution $\mathrm{F}(\eta)$ and its derivatives. Such an assumption is made because we are studying the linear stability of the basic flow $\mathrm{F}(\eta)$ so that the disturbance $\mathrm{g}(\eta)$ is small, $\gamma$ being the growth (or decay) rate of the disturbance.

Substituting (22) in (18) and linearizing, we get

$$
g^{\prime \prime \prime}+F g^{\prime \prime}+\left(\gamma-2 F^{\prime}-M^{2}\right) g^{\prime}+F^{\prime \prime} g=0 \text {. }
$$

Here prime denotes derivative with respect to $\eta$. The boundary conditions for $\mathrm{g}(\eta)$ follow from (13), (21) and (22) as

$$
g(0)=0, g^{\prime}(0)=0, g^{\prime}(\infty)=0 .
$$

Clearly the homogeneous linear Equation (23) subject to the homogeneous boundary conditions (24) constitutes an eigenvalue problem with $\gamma$ as the eigenvalue. Without loss of generality, we can take $g^{\prime \prime}(0)=1$. Solutions of (23) and (24) give an infinite set of eigenvalues $\gamma_{1}<\gamma_{2}<\gamma_{3}$ $<\ldots$ If the smallest eigenvalue $\gamma_{1}$ is negative, then there is an initial growth of disturbances and the flow is unstable. On the other hand, when $\gamma_{1}$ is positive, there is an initial decay and the flow is stable.

Figure 6 shows the variation of the smallest eigenvalue $\gamma_{1}$ for the monotonic solutions (corresponding to upper curves in Figure 2) with b for several values of the magnetic parameter $M$. Since $\gamma_{1}$ is real and positive for these curves, it follows that the monotonic solutions are linearly stable. Further, for a given value of $b, \gamma_{1}$ increases with increase in $M$. Thus we may say that for these stable solutions, disturbances decay more quickly with increase in $M$.

On the other hand, Figure 7 gives a plot of the variation of the lowest eigenvalue $\gamma_{1}$ for the non-monotonic

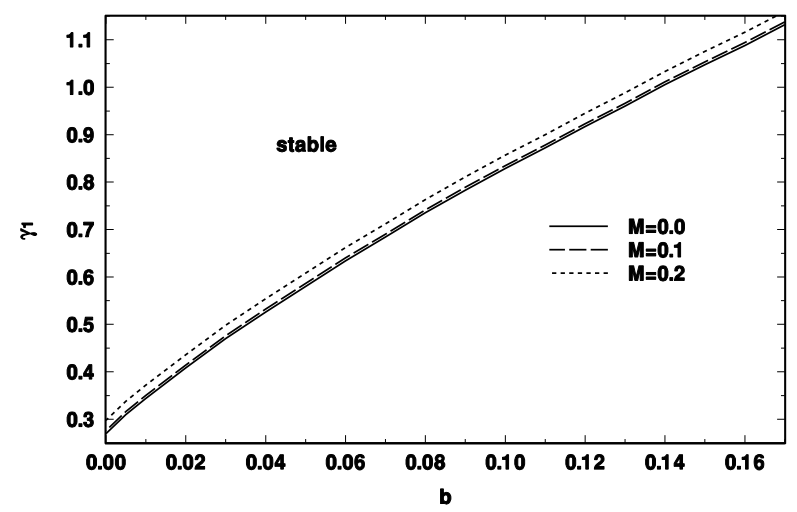

Figure 6. Plot of lowest eigenvalues $\gamma_{1}$ as a function of $b$ for the upper branch solution with different values of $M$. 


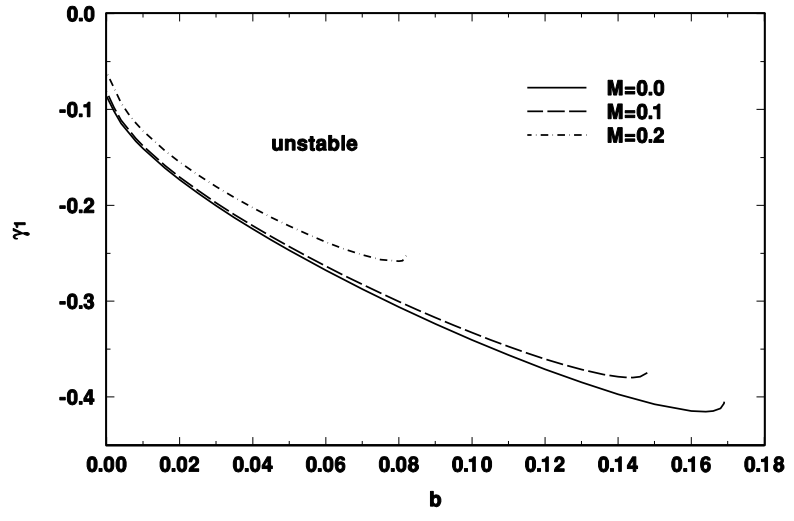

Figure 7. Plot of lowest eigenvalues $\gamma_{1}$ as a function of $b$ for the lower branch solution with different values of M.

solutions (corresponding to lower curves in Figure 2) with $\mathrm{b}$ for several values of $M$. Since $\gamma_{1}<0$ for these curves, it is clear that the non-monotonic solutions are linearly unstable. Further for a given $b,\left|\gamma_{1}\right|$ decreases with increase in $M$ for the non-monotonic solutions. Hence it follows that the growth rate of unstable disturbances decreases with increase in $M$. A physical interpretation of these results is that the magnetic field has a stabilizing influence on the flow. In fact a magnetic field exerts something like a viscous drag on an electrically conducting fluid and also imparts to it some degree of rigidity. Thus magnetic field can be expected to inhibit any tendency to instability. Further from an energetic point of view, a magnetic field is stabilizing since in addition to the dissipation of energy by viscosity, there is dissipation of magnetic energy in the form of Joule heating [12]. Specially, when an electrically conducting fluid is perturbed in the presence of a magnetic field, the disturbances induced by currents, whose energy is dissipated by Joule heating.

It may be noted that the result that the monotonic solution is linearly stable and the non-monotonic solution is linearly unstable in the case of an electrically nonconducting fluid with $M=0$ was arrived at earlier in [8]. Our present stability analysis is based on the approach in [8].

\section{Conclusions}

An investigation is made of steady two dimensional MHD stagnation-point flow of an electrically conducting incompressible viscous fluid over a stretching surface in the presence of a uniform transverse magnetic field. The surface is stretched in its own plane with a velocity proportional to the distance from the stagnation-point. The flow problem is governed by the dimensionless parameter $b$ representing the ratio of the strain-rate of the stagnation flow to that of the stretching surface and the magnetic parameter $M$. It is shown that in addition to the monotonic increasing solution (which is unique) for $b>$ 1 earlier found by Mahapatra and Gupta [7], there exist two solutions in the range $0<b<b_{c}$, where $b_{c}(<1)$ depends on $M$. In this range one solution is monotonic increasing whilst the dual solution is non-monotonic. It is also found numerically that $b_{c}$ decreases with increase in $M$ and when $M>0.23919$, the dual solution does not exist.

A linear stability analysis of the two solutions in the range $0<b<b_{c}$ reveals that the monotonic decreasing solution is linearly stable but the non-monotonic solution is linearly unstable. Further the magnetic field exerts a stabilizing influence on the flow.

\section{Acknowledgements}

We thank the referee for his useful comments which enabled us in presenting an improved version of the paper. The work of one of the authors (T.R.M) is supported under SAP (DRS PHASE II) program of UGC, New Delhi. One of the authors (A.S.G) acknowledges the financial support of Indian National Science Academy, New Delhi for carrying out this work.

\section{References}

[1] L. J. Crane, "Flow Past a Stretching Plate," Zeitschrift für Angewandte Mathematik und Physik, Vol. 21, 1970, pp. 645-647.

[2] K. B. Pavlov, "Magnetohydrodynamic Flow of an Incompressible Viscous Fluid Caused by the Deformation of a Plane Surface," Magnitnaya Gidrodinamika, Vol. 4, 1974, pp. 146-147.

[3] A. Chakrabarti and A. S. Gupta, "Hydromagnetic Flow and Heat Transfer over a Stretching Sheet," Quarterly of Applied Mathematics, Vol. 37, April 1979, pp. 73-78.

[4] H. I. Andersson, "MHD Flow of a Viscoelastic Fluid Past a Stretching Surface," Acta Mechanica, Vol. 95, 1992, pp. 227-230.

[5] T. C. Chiam, "Stagnation-point Flow towards a Stretching Plate," Journal of the Physical Society of Japan, Vol. 63, 1994, pp. 2443- 2444.

[6] T. R. Mahapatra and A. S. Gupta, "Heat Transfer in Stagnation-point Flow towards a Stretching Sheet," Heat Mass Transfer, Vol. 38, 2002, pp. 517-521.

[7] T. R. Mahapatra and A. S. Gupta, "Magnetohydrodynamic Stagnation-point Flow towards a Stretching Sheet," Acta Mechanica, Vol. 152, 2001, pp. 191-196.

[8] J. Paullet and P. Weidman, "Analysis of Stagnation Point Flow towards a Stretching Sheet," International Journal of Non-Linear Mechanics, Vol. 42, No. 9, 2007, pp. 1084-1091.

[9] T. R. Mahapatra, S. K. Nandy, and A. S. Gupta, "Magnetohydrodynamic Stagnation-point Flow of a Power-law Fluid towards a Stretching Surface," International Jour- 
nal of Non-Linear Mechanics, Vol. 44, 2009, pp. 123-128.

[10] J. A. Shercliff, "A Textbook of Magnetohydrodynamics," Pergamon Press, Oxford, 1965.

[11] J. H. Merkin, "Mixed Convection Boundary Layer Flow on a Vertical Surface in a Saturated Porous Medium,"
Journal of Engineering Mathematics, Vol. 14, 1980, pp. 301-313.

[12] S. Chandrasekhar, "Hydrodynamic and Hydromagnetic Stability," Clarendon Press, Oxford, 1961. 